\title{
Vibration Control for a Coupled Pitch- roll Ship Model via a Negative Cubic Velocity Feedback Control
}

\author{
Y. A. Amer ${ }^{\text {a }}$, Taher A. Bahnasy ${ }^{\mathrm{b}, *}$, and Ashraf M. Elmhalawy ${ }^{\mathrm{b}}$ \\ ${ }^{a}$ Department of Mathematics, Faculty of Science, Zagazig University, Zagazig, Egypt. \\ b Physics and Engineering Mathematics Department, Faculty of Engineering, Tanta University, Tanta, Egypt. \\ *Corresponding Author, e-mail: Taher.Bahnasy@f-eng.tanta.edu.eg
}

\begin{abstract}
One of the most essential ship reactions to waves is roll motion. Due to the intricacy of ship wave interactions and their sensitivity, predicting such a reaction is extremely challenging. Because vibration motion is an undesirable occurrence, it must be removed, decreased, or controlled. A coupled Pitch- roll ship model with negative cubic velocity feedback control subjected to parametric excitations is premeditated and solved in this paper. The method of multiple time scales is applied to scrutinize the response of the two modes of the system neighbouring the simultaneous sub-harmonic, and internal resonance situation. Besides, the steady-state solution is determined through the RungKutta Method (RKM) of fourth order. Stability of the steady state solution near this resonance case is discussed and studied applying Lyapunov's first indirect method and Routh- Hurwitz criterion. The influences of the different parameters on the steady state solution are reconnoitred and discussed. The controller effects on the stability are clarified. Simulation results are accomplished with the help of MATLAB and Maple software programs.
\end{abstract}

Keywords: Resonance, active feedback controller, stability analysis.

\section{INTRODUCTION}

After 1863, Froude [1] widely studied the coupled pitchroll ship model, writing that ships have unwanted roll features when the usual pitch frequency is indeed almost doubled the natural frequency of roll ships, three types of displacement (heave, sway or float, and surge) and three angular motions (yaw, pitch, and roll) can be involved, as seen in Figure 1. The general equations of motion are defined by either the Lagrange equation (see, for instance, [2-5]) or Newton's second law of motion (see, for instance, [6-8]).

One of the basic necessities of ship construction is stability against tipping of heavy seas. Capsizing is interrelated with the ship and waves' intense motion. The roll swaying is the most extreme motion of the six ship movements that can result in the ship capsizing. The reaction of ships can be designated by a linear equation for small angles of roll movements. However, as the oscillation amplitude increases, nonlinear effects are inherited. Nonlinearity may spread minor excitation deviations to the point that capsizing is subsidised by the restoring power. The non - linearity is subject to the reconfigured nature of the moments and damping. The ecological loadings are non-linear and beyond the designer's control. Nonlinearity of the moment of reconstruction is based on the form of the diagram of the right arm.
M. A. Abkowitz [9] presented a significant improvement in the forces that function on wave, sway, and yaw motions for the ship. He used the hydrodynamic powers of the Taylor series to extend the direction of forward movement. The formulation has contributed to an infinite set of parameters and can simulate powers to an arbitrary degree of accuracy. Linear and formal non - linearity motion equations can then be generalised. Abkowitz [10, 11], Hwang [12], and Kallstrom and Astrom [13] offered separate methodologies later to approximate the correlation coefficient of such models. Son and Nomoto [14] extended Abkowitz's [9] analysis to incorporate the motion of ship-roll while deriving the forces and moments acting on the ship. Ross [15] created the dynamical differential equations of a ship manoeuvrability across waves utilising Kirchhoff's convolution integral methodology of the mass attached [16]. Kirchhoff's formulas are a series of interactions that are used to interpret the motion formulas from the derivatives of the kinetic energy scheme. They are specific circumstances of Euler-Lagrange formulations. The consequential equations also provide, as in Miline-thomson, and Fossen [17, 18], the Coriolis and centripetal powers.

Rong [19] proposed several of the challenges of weak and high non-linearity sea loads on floating maritime networks. Using a first order multiple-time scale method, including the basic explanation for the saturation phenomenon of ship motion, Nayfeh and Mook [20, 21] made a significant contribution to nonlinear dynamics. Pan et al. [22] reviewed the vibrating behaviours of the mathematical model for a pitch-roll ship with dynamic perturbation using the averaging procedure and two variations of the multi-scale method. For computational simulations, they found that all three theoretical findings were in good harmony. A modification to the saturation phenomenon was however, introduced by both strategies. They extended their research to the case of amplitude modulation sine wave perturbation for the same model in their other paper [23], focusing on the influence of modification on the device's performance and the stability of the algorithm with zero magnitude roll mode. Kamel [24] and Zhou et al. [25] suggested stability and bifurcation methodology through analytical and empirical means, respectively, in consideration of the combination resonance in the presence of internal resonance for the same model that describes the nonlinear partnered pitch-roll motion of ships under sinusoidal harmonic excitation. In other words, their 
analysis centred specifically on the continuity of the twodegree theory of freedom in the periodic solutions and the characteristics of local bifurcations.

A nonlinear relating internal resonance analysis for shear roll and practical pitch output of a spar-specific framework through waves and vortex-induced packing was suggested by Li Wei et al. [26], where even the heave, roll and pitch frequency ratio is about $2: 1: 1$. For the long - span mechanism within its control of first wave loads in the heave and pitch, and spiral driven loads in the roll, with respect to vastly differing wet terrain, the 3 DOFs structure of non - linearity formulas is created.

In Lihua Liang et al. [27], a ride control system (RCS) based on linear quadratic regulator (LQR) and genetic algorithm (GA) functionality is proposed to minimise wave penetrating catamarans (WPC) heave, roll and pitch modulation in beam waves ( 3 degrees of freedom geometry (3 DOF motion)). For the WPC vessel, a robust 3 DOF ride regulation model is expected, consisting of the coupling and separating interactions between spatial and transverse motion. And the complicated coefficients of hydrodynamics and disturbances caused by beam waves are being scrutinised. In addition, in the intermittent flapping procedure, the system is fitted with two stern flaps. In the controller architectural design, the GA approach-based LQR technique is used to greatly reduce the ship's 3 DOF motion. Weighting categories may be gathered to determine the optimal gain, based on the robust search process and the global optimum of GA.

Liang et al. [28, 29], incorporated the 2-degrees of the principle of free movement (heave and pitch) of the Wave Piercing Catamarans (WPC) vessel and proposed for the ride control system (RCS) of the ship dependent on predictive and linear quadratic regulator (LQR) control approaches. Horizontal high-speed vibration elevator induced by rail roughness is solved by shunxin cao et al., A 6-degree of freedom, the model of horizontal vibration of the high-speed elevator car scheme is advanced and premeditated by taking into account the limitations of the active guide shoe's stroke and actuator strength. To decrease the vibration of the car structure, the $\mathrm{H} 2$ standard is chosen. By incorporating the Nonlinear Integral Positive Position Feedback (NIPPF) to control the vibrating system, the formulation of a combined Rayleigh-Van der Pol-Duffing oscillator was modified by Amer et al. [30]. A 3-degree-of - freedom (3-DOF) comprising the cubic nonlinear term and the external force is posed to the system. They supposed to apply the Multiple Scales Method (MSM) to really get the solution from the first approximation.

N. A. Saeed et al. [31] investigated and controlled the nonlinear dynamical behaviors of a nonlinear asymmetric rotating shaft system. They introduced a linear proportionalderivative controller to suppress the lateral vibrations of the rotating shaft. Perturbation and numerical solution for vibrations analysis and controlling of a system which simulates the vibrations of a nonlinear composite beam model were discussed by M. sayed et al. [32]. The stability analysis of the system were obtained by frequency response (FR). They conducted Bifurcation analysis using various control parameters such as natural frequency, detuning parameter, feedback signal gain, control signal gain, and other parameters.

The nonlinear vibrations of a contact-mode atomic force microscopy (AFM) model subjected to multi excitations are controlled via a time-delayed positive position feedback (PPF) controller by Y.S. Hamed et al. [33]. To obtain the approximate nonlinear dynamical behaviour of the AFM system they applied the multiple time scales perturbation method. In addition, Y.S. Hamed et al. [34] investigated the nonlinear oscillations, stability and resonance of a cantilever beam system which carrying an intermediate lumped mass. The response of amplitudes and phases of the differential equation governing is derived applying the multiple scale perturbation (MSP) method. The nonlinear vibration control is analyzed using a new nonlinear modified positive position feedback (MPPF) approach. The nonlinear approximate solutions for this system up to and inclusive the second order approximation are solved.

The aim of this research is to change the Xinye Li et al. [30] model by introducing a negative feedback controller for the cubic velocity signal. A 2-DOF device subjected to multi parametric excitations is described by this model. In order to achieve the semi-closed solution, MTSM is used to analyse the reaction of the modified device near to the synchronised internal and sub-harmonic resonance situation. The system's stability is explored by applying frequency response equations close this worst simultaneous resonance event. Any guidelines are recorded about the various device parameters and the effects of the controller on the actions of the device are provided numerically. Comparisons are stated with the numerical solution.

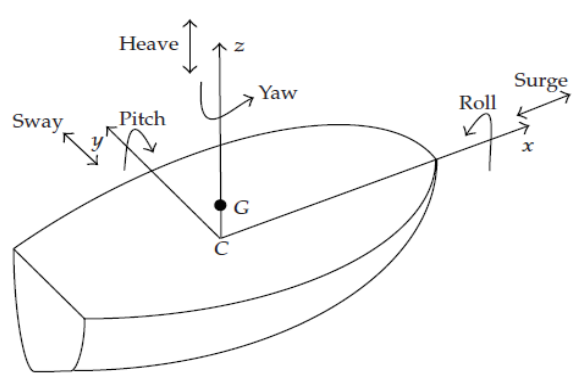

Fig. 1. Ship simulation model presenting the 6-degrees of freedom.

\section{SYSTEM MODEL AND MATHEMATICAL ANALYSIS}

The mathematical model for the system is given in [30] as:

$$
\begin{aligned}
& \ddot{U}(t)+\omega_{1}^{2} U(t)=\varepsilon\left(\alpha_{1} U(t) V(t)\right. \\
& \left.+\alpha_{2} U(t) \dot{V}^{2}(t)-\mu_{1} \dot{U}(t)\right), \\
& \ddot{V}(t)+\omega_{2}^{2} V(t)=\varepsilon\left(\delta_{1} U^{2}(t)+F \cos (\Omega t)-\mu_{2} V\right),
\end{aligned}
$$

where $U(t)$ and $V(t)$ denote the roll and pitch orientations respectively, $\Omega$ is the encounter frequency, $\omega_{1}, \omega_{2}$ are internal frequencies, and $\varepsilon$ is a small perturbation parameter. Considering external parametric pitch and roll model excitation forces $\left(F_{1}, F_{2}\right)$, and the impact of active 
control of negative cubic velocity feedback be discussed. With this considerations system (1) will be:

$\ddot{U}(t)+\omega_{1}^{2} U(t)=\varepsilon\left(\alpha_{1} U(t) V(t)+\alpha_{2} U(t) \dot{V}^{2}(t)\right.$

$\left.-\mu_{1} \dot{U}(t)+F_{1} U(t) \cos \left(\Omega_{1} t\right)-G_{1} \dot{U}^{3}\right)$,

$\ddot{V}(t)+\omega_{2}^{2} V(t)=\varepsilon\left(\delta_{1} U^{2}(t)+F_{2} \cos \left(\Omega_{2} t\right)-\mu_{2} \dot{V}\right)$,

The second order approximation is given by applying the Multiple Time Scales (MTSM) procedure, so $U(t)$ and $V(t)$ in power series form will be:

$U\left(T_{o}, T_{1}, \varepsilon\right)=U_{o}\left(T_{o}, T_{1}\right)+\varepsilon U_{1}\left(T_{o}, T_{1}\right)+O\left(\varepsilon^{2}\right)$,

$V\left(T_{o}, T_{1}, \varepsilon\right)=V_{o}\left(T_{o}, T_{1}\right)+\varepsilon V_{1}\left(T_{o}, T_{1}\right)+O\left(\varepsilon^{2}\right)$.

where the time derivative takes the values:

$\frac{d}{d t}=D_{0}+\varepsilon D_{1}+O\left(\varepsilon^{2}\right), \frac{d^{2}}{d t^{2}}=D_{o}^{2}+2 \varepsilon D_{0} D_{1}+O\left(\varepsilon^{2}\right)$,

and $T_{n}=\varepsilon^{n} t, D_{n}=\frac{\partial}{\partial T_{n}}, n=0,1$.

Switching equations (3), (4) into (2) and equating same power of $\varepsilon$ coefficients to take out:

$O\left(\varepsilon^{0}\right)$ :

$\left(D_{o}^{2}+\omega_{o}^{2}\right) U_{0}=0$,

$\left(D_{o}^{2}+\omega_{o}^{2}\right) V_{0}=0$.

$O(\varepsilon)$ :

$\left(D_{o}^{2}+\omega_{1}^{2}\right) U_{1}=-2 D_{o} D_{1} U_{o}-\mu_{1} D_{o} U_{o}+F_{1} U_{o} \cos \left(\Omega_{1} t\right)$

$+\alpha_{1} U_{o} V_{o}+\alpha_{2} U_{o}\left(D_{o} V_{o}\right)^{2}-G_{1}\left(D_{o} U_{o}\right)^{3}$

$\left(D_{o}^{2}+\omega_{2}^{2}\right) V_{1}=-2 D_{o} D_{1} V_{o}-\mu_{2} D_{o} U_{o}$

$+F_{2} V_{o} \cos \left(\Omega_{2} t\right)+\delta_{1} U_{o}^{2}$.

Obviously solution of (5) is:

$U_{0}=A_{1}\left(T_{1}\right) e^{i \omega_{1} T_{0}}+\overline{A_{1}}\left(T_{1}\right) e^{-i \omega_{1} T_{0}}$,

$V_{0}=B_{1}\left(T_{1}\right) e^{i \omega_{2} T_{0}}+\bar{B}_{1}\left(T_{1}\right) e^{-i \omega_{2} T_{0}}$.

Substituting (8) for (6) and (7), we then have:

$\left(D_{o}^{2}+\omega_{1}^{2}\right) U_{1}=-2\left[i \omega_{1} D_{1} A_{1} e^{i \omega_{1} T_{o}}-i \omega_{1} D_{1} \overline{A_{1}} e^{-i \omega_{1} T_{o}}\right]$

$-\mu_{1}\left[i \omega_{1} A_{1} e^{i \omega_{1} T_{o}}-i \omega_{1} \overline{A_{1}} e^{-i \omega_{1} T_{o}}\right]+$

$+F_{1} \cos \left(\Omega_{1} T_{o}\right)\left[A_{1} e^{i \omega_{1} T_{o}}+\overline{A_{1}} e^{-i \omega_{1} T_{o}}\right]$

$+\alpha_{1}\left[A_{1} e^{i \omega_{1} T_{o}}+\overline{A_{1}} e^{-i \omega_{1} T_{o}}\right]\left[B_{1} e^{i \omega_{2} T_{o}}+\bar{B}_{1} e^{-i \omega_{2} T_{o}}\right]$

$+\alpha_{2}\left[A_{1} e^{i \omega_{1} T_{o}}+\overline{A_{1}} e^{-i \omega_{1} T_{o}}\right]\left(i \omega_{2} B_{1} e^{i \omega_{2} T_{o}}-i \omega_{2} \overline{B_{1}} e^{-i \omega_{2} T_{o}}\right)^{2}$

$-G_{1}\left(i \omega_{1} A_{1} e^{i \omega_{1} T_{o}}-i \omega_{1} \overline{A_{1}} e^{-i \omega_{1} T_{o}}\right)^{3}$,

$\left(D_{o}^{2}+\omega_{2}^{2}\right) V_{1}=-2\left[i \omega_{2} D_{1} B_{1} e^{i \omega_{2} T_{o}}-i \omega_{2} D_{1} \bar{B}_{1} e^{-i \omega_{2} T_{o}}\right]$

$-\mu_{2}\left[i \omega_{2} B_{1} e^{i \omega_{2} T_{o}}-i \omega_{2} \bar{B}_{1} e^{-i \omega_{2} T_{o}}\right]$

$+\delta_{1}\left[A_{1}^{2} e^{2 i \omega_{1} T_{o}}+\bar{A}_{1}^{2} e^{-2 i \omega_{1} T_{o}}+2 A_{1} \overline{A_{1}}\right]$

$+F_{2} \cos \left(\Omega_{2} T_{o}\right)\left[B_{1} e^{i \omega_{2} T_{o}}+\bar{B}_{1} e^{-i \omega_{2} T_{o}}\right]$.
Now, due to resonance case, we will study the system's severe operating approaches, this worst case at:

$\Omega_{1}=2 \omega_{1}+\varepsilon \sigma_{1}$,

$\omega_{2}=2 \omega_{1}+\varepsilon \sigma_{2}$.

Eliminating coefficients of all secular terms from (9) and (10), yields

$-i \omega_{1} \mu_{1} A_{1}-2 i \omega_{1} D_{1} A_{1}+\alpha_{1} \overline{A_{1}} B_{1} e^{i \sigma_{2} T_{1}}$

$+\alpha_{2} A_{1} B_{1} \bar{B}_{1} \omega_{2}^{2}-3 i G_{1} \omega_{1}^{3} A_{1}^{2} \overline{A_{1}}+\frac{f \overline{A_{1}}}{2} e^{i \sigma_{1} T_{1}}=0$,

$-i \omega_{2} \mu_{2} B_{1}-2 i \omega_{2} D_{1} B_{1}+\delta_{1} A_{1}^{2} e^{-i \sigma_{2} T_{1}}=0$.

Converting the functions $A_{1}$ and $B_{1}$ into the polar form:

$A_{1}=\frac{a_{1}}{2} e^{i \beta_{1}}$,

$B_{1}=\frac{a_{2}}{2} e^{i \beta_{2}}$,

where $a_{1}, a_{2}$ are the system amplitude and $\beta_{1}, \beta_{2}$ are the system phase.

Introducing (14) into (12), (13), in the resulting equations, we compare the real and imaginary parts, then we get:

$\dot{a}_{1}=-\frac{\mu_{1} a_{1}}{2}+\frac{\alpha_{1} a_{1} a_{2} \sin \left(\theta_{2}\right)}{4 \omega_{1}}-\frac{3 G_{1} \omega_{1}^{2} a_{1}^{3}}{8}+\frac{F_{1} a_{1} \sin \left(\theta_{1}\right)}{4 \omega_{1}}$,

$a_{1} \dot{\theta}_{1}=\sigma_{1} a_{1}+\frac{\alpha_{1} a_{1} a_{2} \cos \left(\theta_{2}\right)}{2 \omega_{1}}+\frac{\alpha_{2} a_{1} a_{2}^{2} \omega_{2}^{2}}{4 \omega_{1}}+\frac{F_{1} a_{1} \cos \left(\theta_{1}\right)}{2 \omega_{1}}$,

$\dot{a}_{2}=-\frac{\mu_{2} a_{2}}{2}-\frac{\delta_{1} a_{1}^{2} \sin \left(\theta_{2}\right)}{4 \omega_{1}}$,

$a_{2} \dot{\theta}_{2}=\sigma_{2} a_{2}+\frac{\alpha_{1} a_{2}^{2} \cos \left(\theta_{2}\right)}{2 \omega_{1}}+\frac{\alpha_{2} a_{2}^{3} \omega_{2}^{2}}{4 \omega_{1}}$

$+\frac{F_{1} a_{2} \cos \left(\theta_{1}\right)}{2 \omega_{1}}-\frac{\delta_{1} a_{1}^{2} \cos \left(\theta_{2}\right)}{4 \omega_{1}}$,

where

$\theta_{1}=\sigma_{1} T_{1}-2 \beta_{1}$,

$\theta_{2}=\sigma_{2} T_{1}+\beta_{2}-2 \beta_{1}$

\section{A. Steady state solution}

The amplitude and phase steady state solution is obtained by substituting

$\dot{a}_{1}=\dot{\theta}_{1}=\dot{a}_{2}=\dot{\theta}_{2}=0$ into Eq. (15)-(18), we get:

$$
\frac{F_{1} a_{1} \cos \left(\theta_{1}\right)}{2 \omega_{1}}=-\left(\frac{\omega_{1} \sigma_{1} a_{1}}{2}+\frac{\alpha_{1} a_{1} a_{2} \cos \left(\theta_{2}\right)}{4}+\frac{\alpha_{2} a_{1} a_{2}^{2} \omega_{2}^{2}}{8}\right) \text {, }
$$

$\frac{F_{1} a_{1}}{4} \sin \left(\theta_{1}\right)=\frac{\omega_{1} \mu_{1} a_{1}}{2}-\frac{\alpha_{1} a_{1} a_{2} \sin \left(\theta_{2}\right)}{4}+\frac{3 G_{1} \omega_{1}^{3} a_{1}^{3}}{8}$, 
$\sin \left(\theta_{2}\right)=-\frac{2 \omega_{2} \mu_{2} a_{2}}{\delta_{1} a_{1}^{2}}$,
$\cos \left(\theta_{2}\right)=\frac{4 \omega_{2}\left(\sigma_{2}-\sigma_{2}\right) a_{2}}{\delta_{1} a_{1}^{2}}$.

Squaring and adding both sides of (21) and (22), gives

$a_{2}^{2}=\frac{\delta_{1} a_{1}^{4}}{4 \omega_{2}^{2}\left[4\left(\sigma_{2}-\sigma_{1}\right)^{2}+\mu_{2}^{2}\right]}$.

Substituting from (22)-(24) into (20) and (21), squaring and adding the resultant equations to have the frequency response equation in the form:

$$
\begin{aligned}
& \Gamma_{2}^{2} a_{1}^{8}+2 \Gamma_{1} \Gamma_{2} a_{1}^{6}+\left(\Gamma_{1}^{2}+\Gamma_{3}^{2}+\Gamma_{2} \omega_{1} \sigma_{1}\right) a_{1}^{4} \\
& +\left(\Gamma_{1} \omega_{1} \sigma_{1}+\Gamma_{3} \omega_{1} \mu_{1}\right) a_{1}^{2}+\frac{\omega_{1}^{2}}{4}\left(\mu_{1}^{2}+\sigma_{1}^{2}\right)-\frac{F_{1}^{2}}{16}=0,
\end{aligned}
$$

where

$$
\begin{aligned}
& \Gamma_{1}=\frac{\alpha_{1} \delta_{1}\left(\sigma_{2}-\sigma_{1}\right)}{4 \omega_{2}\left[4\left(\sigma_{2}-\sigma_{1}\right)^{2}+\mu_{2}^{2}\right]}, \\
& \Gamma_{2}=\frac{\alpha_{2} \delta_{1}^{2}}{32\left[4\left(\sigma_{2}-\sigma_{1}\right)^{2}+\mu_{2}^{2}\right]}, \\
& \Gamma_{3}=\frac{\alpha_{1} \delta_{1} \mu_{2}}{8 \omega_{2}\left[4\left(\sigma_{2}-\sigma_{1}\right)^{2}+\mu_{2}^{2}\right]}+\frac{3 G_{1} \omega_{1}^{3}}{8} .
\end{aligned}
$$

\section{B. Stability analysis}

Linearizing equations (15)-(18) according to the Lyapunov first (indirect) form, given in Soltine et al. [35], Liang [36] to address the stability behaviour of these solutions, to give the following system:

$$
\left[\begin{array}{c}
\dot{a}_{1} \\
\dot{\theta}_{1} \\
\dot{a}_{2} \\
\dot{\theta}_{2}
\end{array}\right]=\left[\begin{array}{llll}
\xi_{11} & \xi_{12} & \xi_{13} & \xi_{14} \\
\xi_{21} & \xi_{22} & \xi_{23} & \xi_{24} \\
\xi_{31} & \xi_{32} & \xi_{33} & \xi_{34} \\
\xi_{41} & \xi_{42} & \xi_{43} & \xi_{44}
\end{array}\right]\left[\begin{array}{c}
a_{1} \\
\theta_{1} \\
a_{2} \\
\theta_{2}
\end{array}\right],
$$

where $\chi_{i j},\{i, j=1,2,3,4\}$ are included in "appendix".

The stability of the steady-state solution therefore, depends on the Jacobian matrix's eigenvalues that can be obtained by solving the following characteristic equation:

$$
\left|\begin{array}{cccc}
\xi_{11}-\lambda & \xi_{12} & \xi_{13} & \xi_{14} \\
\xi_{21} & \xi_{22}-\lambda & \xi_{23} & \xi_{24} \\
\xi_{31} & \xi_{32} & \xi_{33}-\lambda & \xi_{34} \\
\xi_{41} & \xi_{42} & \xi_{43} & \xi_{44}-\lambda
\end{array}\right|=0
$$

or

$$
\lambda^{4}+\eta_{1} \lambda^{3}+\eta_{2} \lambda^{2}+\eta_{3} \lambda+\eta_{4}=0
$$

where $\lambda$ denotes the Jacobian matrix $[J]$ eigenvalues, $\eta_{k}, k=1: 4$ are the characteristic equation coefficients depending on the parameters $\omega_{1}, \omega_{2}, \alpha_{1}, \alpha_{2}, \mu_{1}, \mu_{2}$, and $\delta_{1}$ given in Appendix. As shown by the Routh-Huriwitz criterion the solution of the state is asymptotically stable if and only if it is: $\eta_{1}>0$

$\eta_{4}>0$,

$\eta_{1} \eta_{2}-\eta_{3}>0$, and

$\eta_{3}\left(\eta_{1} \eta_{2}-\eta_{3}\right)-\eta_{1}^{2} \eta_{4}>0$

Routh-Huriwitz conditions (30) are established numerically with the help of MATLAB software for estimating the stable and the unstable regions in the frequency response curves.

\section{RESULTS AND DISCUSSION}

In this section, we demonstrate the behaviour of the amplitude and phase of the system in the resonance and the non-resonance states. To assess the numerical solution of the given scheme, the procedure of RK4 is being used, supposing the following parameters: $\mu_{1}=0.4, \mu_{2}=1, \delta_{1}=1, \alpha_{1}=1$, $\alpha_{2}=5, F_{1}=15, F_{2}=9.6, G_{1}=4, \omega_{1}=6, \Omega_{2}=13$. The time response is seen in Figure 2 (a) and (b) for the magnitudes $U$, and $V$ respectively at non-resonant situation and without control $\left(G_{1}=0\right)$. Figure 3 (a), (b) shows the time response for the system without and with applying negative cubic velocity feedback active control (i.e. $G_{1}=0, G_{1}=4$ ) with sub-harmonic resonance instance $\Omega_{1}=2 \omega_{1}+\varepsilon \sigma_{1}, \omega_{2}=$ $2 \omega_{1}+\varepsilon \sigma_{2}$. Figure 3 (c), (d) clarifies the system phase plane. Fig. $3(\mathrm{a}, \mathrm{b})$ demonstrated that the amplitude of the system is reduced to about $98 \%$ in $U$ direction and about $99.8 \%$ in $V$ direction under the effect of the negative feedback signal for the cubic velocity which represented by the coefficient of gain $G_{1}$. Liang et al (2017) measure the reduction ratio as (balanced amplitude before submission of control- balanced amplitude after submission of control) / steady amplitude before submission of control).

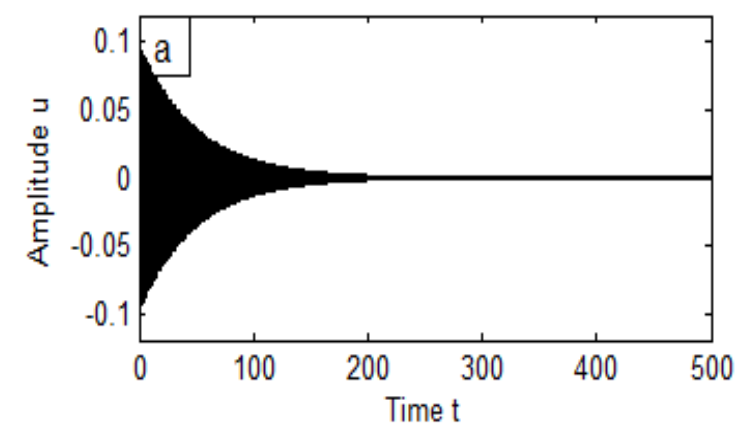

Fig. 2. (a) The time response solution of $u$ at $G_{1}=0$, at none resonance case.

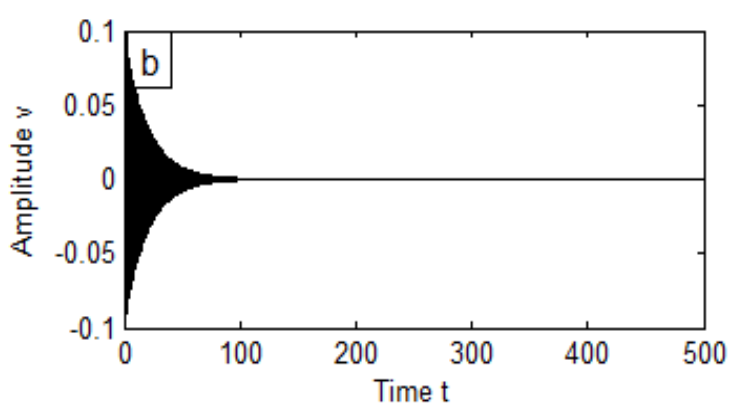

Fig. 2. (b) the time response solution of $v$ at $G_{1}=0$, at none resonance case. 

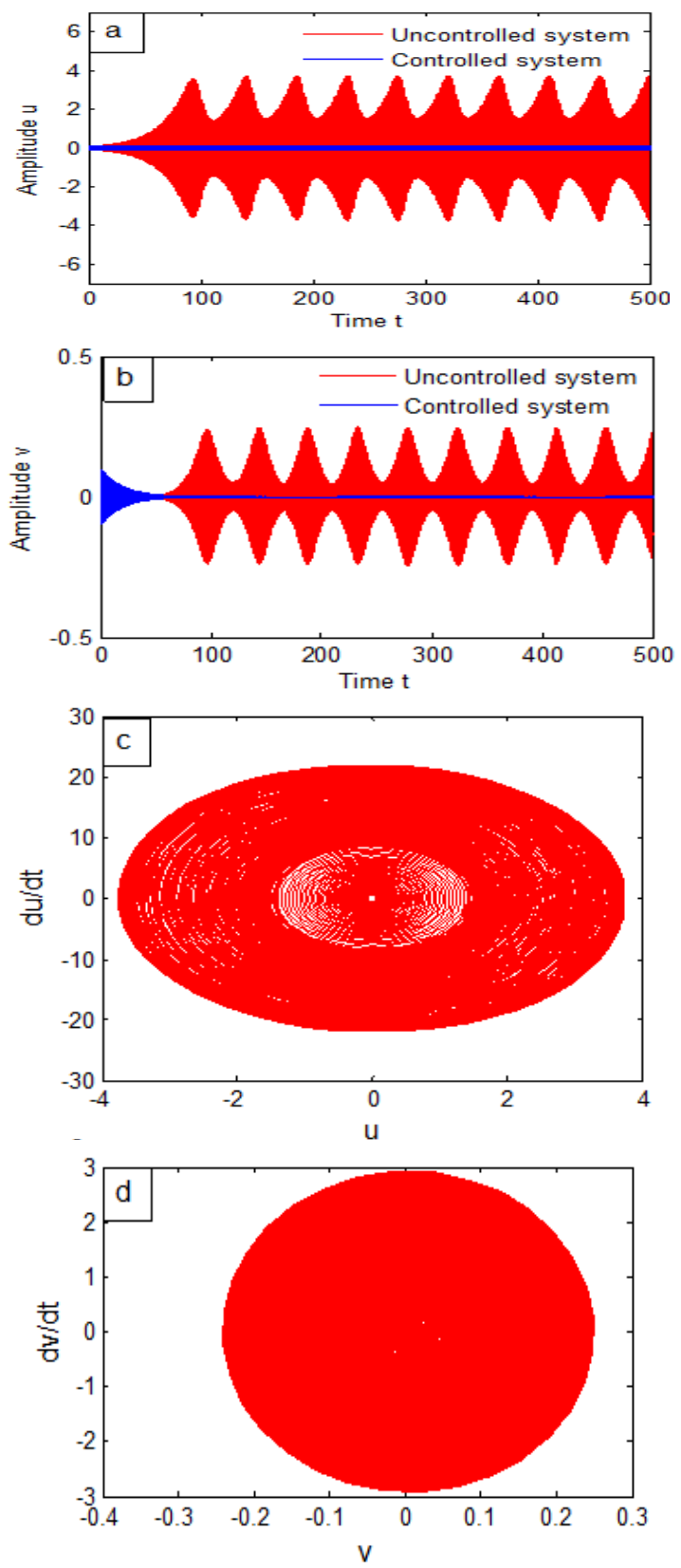

Fig. 3. (a), (b) the time response solution at $G_{1}=0, G_{1}=4$,

(c) and (d) system phase plane.

In Figure 4, for controlled and uncontrolled processes, the response curve is shown. This curve shows that, in the event of an uncontrolled system, the equilibrium area (black curve) is very small compared with the unstable region (red curve). The use of the negative cubic velocity feedback control for the coupled pitch-roll system, as seen in Figure 5, improves the stable area. Figures 6-11 clarify the effects of input parameters on the response curves. We observe in figures 6,7 that system amplitude is proportional inversely with varying the damping parameters $\mu_{1}, \mu_{2}$ without shifting

It is clear from Figure 8 that it increases positively or declines adversely $G_{1}$ reduces the system amplitudes. Modifying the original natural frequency $\omega_{1}$ of the system modes changes the amplitudes in the response curves inversely, as clarified in figures 9,10 , whilst the change of the external force $F_{1}$ leads to a proportional change in system amplitude as illustrated in figure 11 .

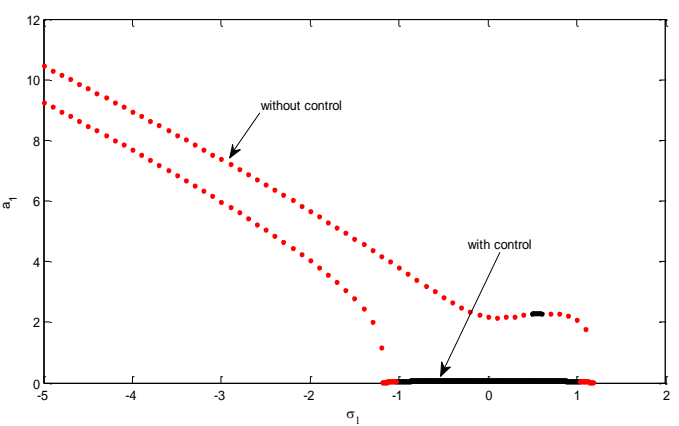

Fig. 4. Response curve for controlled and uncontrolled system, stability region (black curve) and unstable region (red curve).

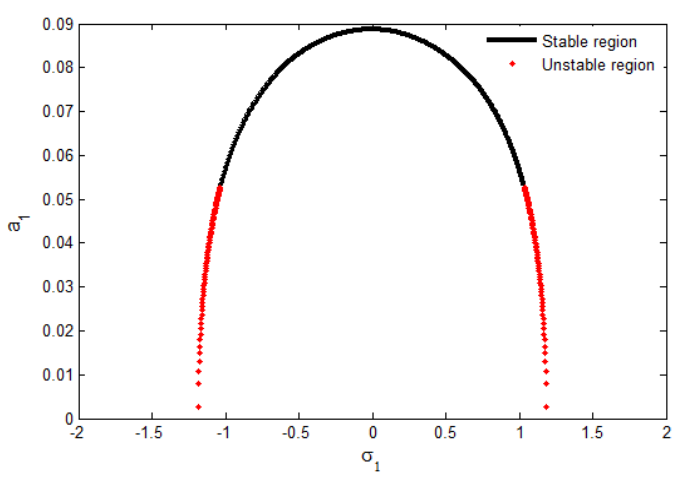

Fig. 5. Response curve for controlled system, stability region (black curve) and unstable region (red curve).

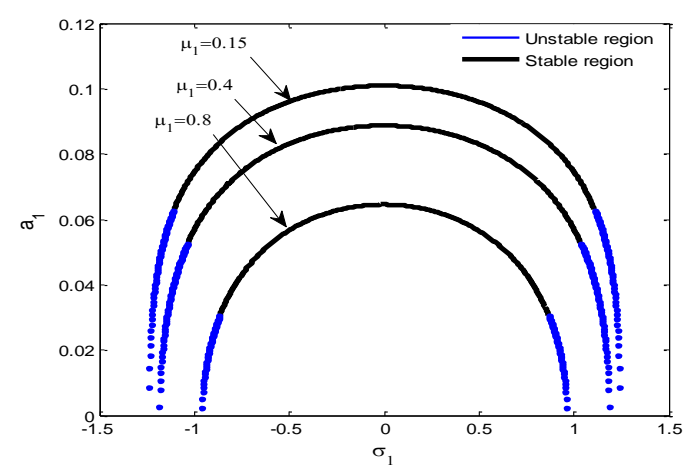

Fig. 6. Response curve $a_{1}$ versus $\sigma_{1}$ for controlled system with various

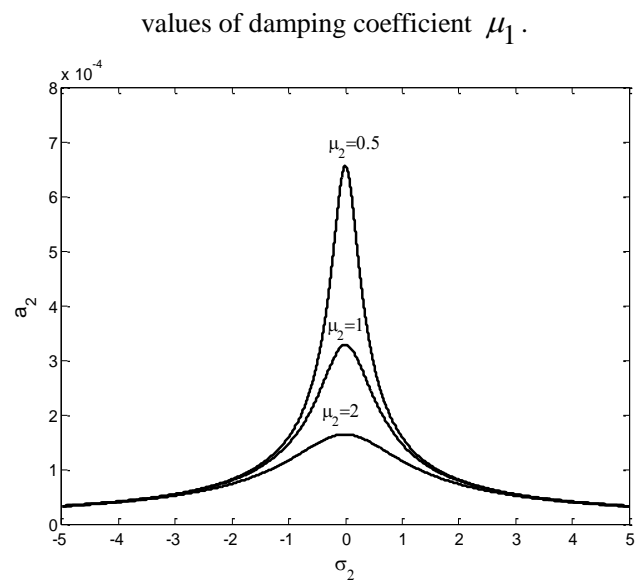

Fig. 7. Response curve $a_{2}$ versus $\sigma_{2}$ for controlled system with various values of damping coefficient $\mu_{2}$. 


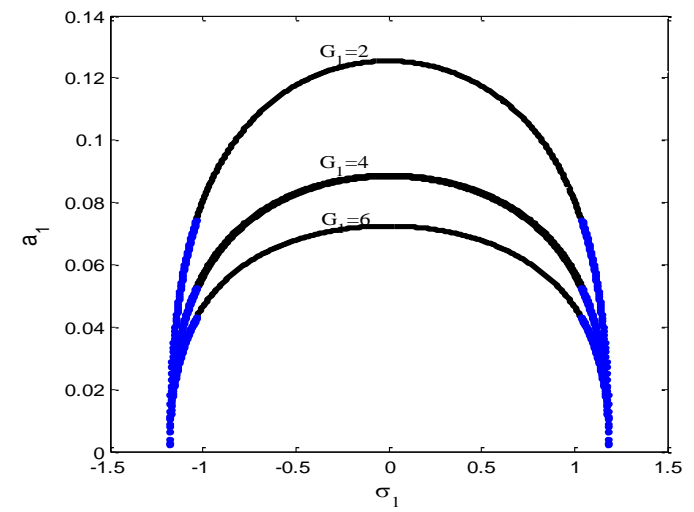

Fig 8. Response curve $a_{1}$ versus $\sigma_{1}$ for controlled system with various values of the control gain $G_{1}$.

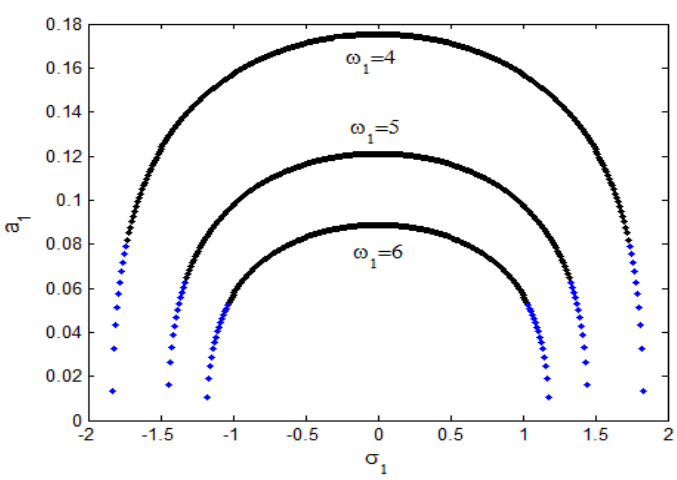

Fig. 9. Response curve $a_{1}$ versus $\sigma_{1}$ for controlled system with various values of $\omega_{1}$

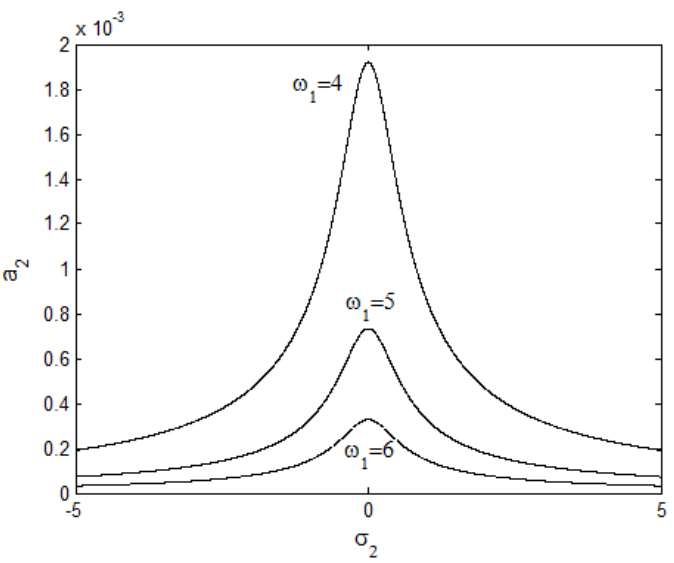

Fig. 10. Response curve $a_{2}$ versus $\sigma_{2}$ for controlled system with various values of $\omega_{1}$.

Both solutions induced by (MTSM) and numerical solution using Rung-Kutta Method (RKM) of fourth order are matched in figures 12-14 for time history and frequency response curves for the system. Figure 12 shows good agreement between the approximate solution (blue curve) and the analytical outcome (red curve) of the resonance case active control scheme equation, and Figures 12 and 14 show good results for the frequency response between the two methods used (MTSM and RKM).

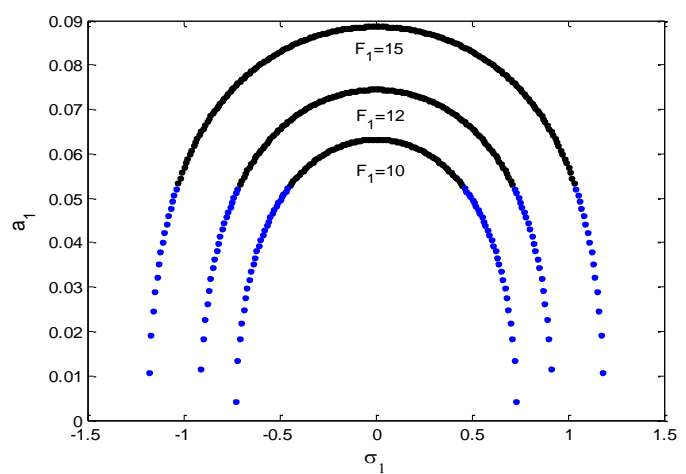

Fig. 11. Response curve $a_{1}$ versus $\sigma_{1}$ for controlled system with various values of the force $F_{1}$.

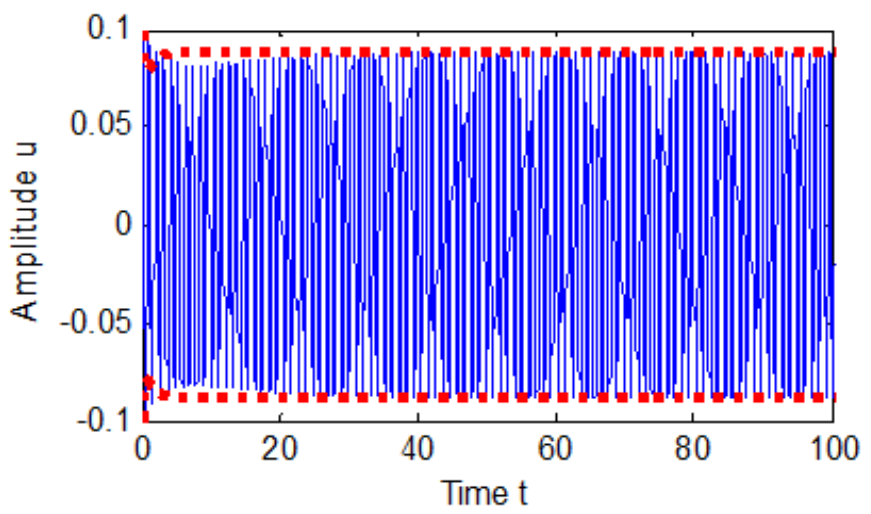

Fig. 12. Comparison between the amplitude induced (MTSM) and (RKM) of fourth order (time history). At resonance case

$\Omega_{1}=2 \omega_{1}+\varepsilon \sigma_{1}, \omega_{2}=2 \omega_{1}+\varepsilon \sigma_{2}$ and with control, $G_{1}=4$.

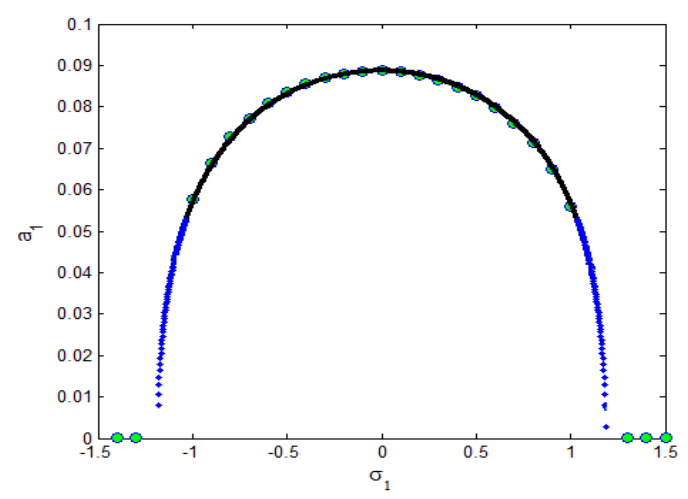

Fig. 13. Comparison between analytic and numerical solutions (response curve $a_{1}$ versus $\left.\sigma_{1}\right)$.

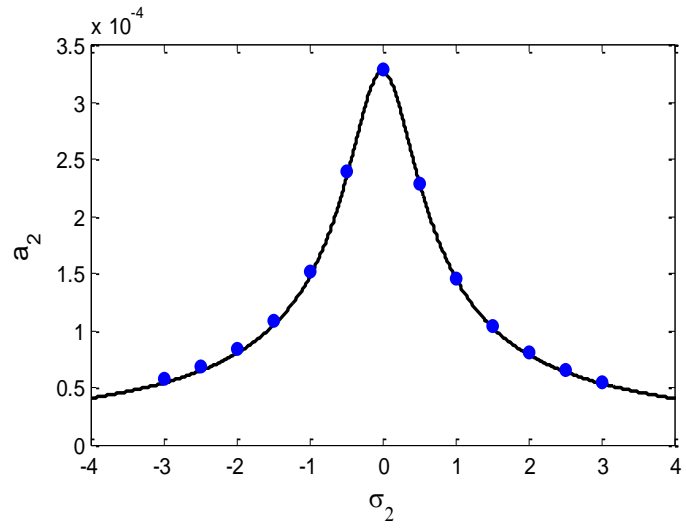

Fig. 14. Comparison between analytic and numerical solutions (response curve $a_{2}$ versus $\sigma_{2}$ ). 


\section{CONCLUSION}

In this research, a coupled pitch-roll model was presented and solved using multiple time scales method and discussed the worst resonance cases of under negative cubic velocity feedback control. This worst resonance cases were instantaneous internal, and sub-harmonic resonance. The effect of negative cubic velocity feedback controller was illustrated and discussed. It was shown that control effect of the first mode was about $98 \%$ and about $99.8 \%$ in the second mode. A desired stability review has also been carried out and appropriate decisions have been found for the feedback benefits in order to reduce the peak amplitude. In addition, analytic solutions were compared with numerical approximation solutions using Rung-Kutta method, these comparisons gave a good agreement between analytic and numerical solutions.

\section{REFERENCES}

[1] Froude W., Remarks on Mr. Scott-Russell's paper on rolling. In The Papers of William Froude, INA (1955) (also Transactions, INA, 4, (1863).

[2] A. H. Nayfeh, D. T. Mook, and L. R. Marshall, "Nonlinear coupling of pitch and roll modes in ship motions," Journal of Hydronautics, vol. 7, no. 4, pp. 145-152 (1973).

[3] A. H. Nayfeh, D. T. Mook, and L. R. Marshall, "Perturbation-energy approach for the development of the nonlinear equations of ship motion," Journal of Hydronautics, vol. 8, no. 4, pp. 130-136 (1974).

[4] A. H. Nayfeh and D. T. Mook, Nonlinear Oscillations, Pure and Applied Mathematics, John Wiley \& Sons, New York, NY, USA (1979).

[5] B. M. Suleiman, Identification of finite-degree-of-freedom models for ship motions, Ph.D. thesis, Virginia Polytechnic Institute and State University, Blacksburg, Va, USA (2000).

[6] E. M. Lewandowski, The Dynamics of Marine Craft Maneuvering and Seakeeping, vol. 2 of Advanced Series on Ocean Engineering,World Scientific, Singapore (2004).

[7] T. Perez, Ship Motion Control; Course Keeping and Roll Stabilization Using Rudder and Fins, Advances in Industrial Control, Springer, Berlin, Germany (2005).

[8] T. I. Fossen, Guidance and Control of Ocean Vehicles, JohnWiley \& Sons, New York, NY, USA (1994).

[9] M. A. Abkowitz, "Lectures on ship hydrodynamics-steering and maneuverability," Tech. Rep. Hy- 5, Hydro- and Aerodynamics Laboratory, Lyngby, Denmark (1964).

[10] M. A. Abkowitz, "System identification techniques for ship maneuvering trials," in Proceedings of the Symposium on Control Theory and Navy Applications, pp. 337-393, Monterey, Calif, USA (1975).

[11] M. A. Abkowitz, "Measurements of hydrodynamic characteristics from ship maneuvering trials by system identification," Transactions of the Society of Architects andMarine Engineering, vol. 88, pp. 283- 318 (1981).

[12] W. Y. Hwang, Application of system identification to ship maneuvering, Ph.D. thesis, Massachusetts Institute of Technology, Cambridge, Mass, USA (1980).

[13] C. G. Ka"llstro"m and K. J. A strom, "Experiences of system identification applied to ship steering," Automatica, vol. 17, no. 1, pp. 187-198 (1981)

[14] K.-H. Son and K. Nomoto, "On the coupled motion of steering and rolling of a high-speed container ship," Naval Architecture and Ocean Engineering, vol. 20, pp. 73-83 (1982).

[15] A. Ross, Nonlinear Manoeuvring Models for Ships: a Lagrangian Approach, Ph.D. thesis, Norwegian University of Science and Technology, Trondheim, Norway (2008).

[16] G. Kirchhoff, Advanced Engineering Mathematics, JohnWiley \& Sons, New York, NY, USA (1869).

[17] L. M. Milne-Thomson, Theoretical Hydrodynamics, MacMillan, London, UK (1968).

[18] T. I. Fossen, Marine Control Systems: Guidance, Navigation and Control of Ships, Rigs and Underwater Vehicles, Marine Cyberentics
AS, Trondheim, Norway (2002)

[19] Z. Rong, Studies of weak and strong nonlinear sea loads on floating marine structures, M.S. thesis, Norges Tekniske Hoegskol, Trodheim, Norway (1994).

[20] Nayfeh, A.H., Mook, D.T.: A saturation phenomenon in the forced response of systems with quadratic nonlinearities. In: Proceedings 8th International Conference on Nonlinear Oscillations, Prague, Czechoslovakia, pp. 511-516 (1978).

[21] Nayfeh, A.H., Mook, D.T.: Nonlinear Oscillations. Wiley, New York (1979)

[22] Pan, R., Davies, H.G.: Response of a nonlinearly coupled pitch-roll ship model under harmonic excitation. Nonlinear Dyn. 9(4), 349-368 (1996).

[23] Pan, R., Davies, H.G.: Non-stationary response of a nonlinearly coupled pitch-roll ship model under modulated excitation. J. Sound Vib. 192(3), 669-699 (1996).

[24] Kamel, M.M.: Bifurcation analysis of a nonlinear coupled pitch-roll ship. Math. Comput. Simul. 73(5), 300-308 (2007).

[25] Zhou, L., Chen, F.: Stability and bifurcation analysis for a model of a nonlinear coupled pitch-roll ship. Math.Comput. Simul. 79(2), 149166 (2008).

[26] LI Wei, Tang You-gang, Liu Li-qin, Li Yan, Wang Bin, Internal Resonances for Heave, Roll and Pitch Modes of A Spar Platform Considering Wave and Vortex-Induced Loads in the Main Roll Resonance, China Ocean Eng., 31(4), 408-417 (2017).

[27] Lihua Liang, Jia YuanID, Songtao Zhang, Hongyu Shi, Yanwen Liu, Peng Zhao, Design ride control system using two stern flaps based 3 DOF motion modeling for wave piercing catamarans with beam seas, Plos One, https://doi.org/10.1371/ March 25, (2019).

[28] Liang LH, Sun MX, Zhang ST, Wen Y. A integrate control system design of WPC with active T-foil and transom stern flap for vertical motion improvement. Journal of Computational Information Systems, 11(9):3217-3227 (2015)

[29] Liang LH, Yuan J, Zhang ST. Application of model predictive control technique for wave piercing catamarans ride control system. IEEE International Conference on Mechatronics and Automation (ICMA). 726-731 (2016).

[30] Amer, Y. A., EL-Sayed, A. T., Abd EL-Salam, M. N., Outcomes of the NIPPF Controller Linked to a Hybrid Rayleigh - Van der PolDuffing Oscillator Appendix, Joutnal of Control Engineering and Applied Informatics, Vol. 22 (3), pp. 33-41,(2020).

[31] N. A. Saeed, S. I. El-Bendary, M. Sayed, M. S. Mohamed, S. K Elagan, On the oscillatory behaviours and rub-impact forces of a horizontally supported asymmetric rotor system under positionvelocity feedback controller, Latin American Journal of Solids and Structures, 18(2), e349, (2021).

[32] M. Sayed, A. A. Mousa, D. Y. Alzaharani, I. H. Mustafa and S. I. ElBendary, Bifurcation analysis of a composite cantilever beam via 1:3 internal resonance, Journal of the Egyptian Mathematical Society, 28-45, https://doi.org/10.1186/s42787-020-00102-7, (2020).

[33] Y.S. Hamed, K.M. Albogamy, M. Sayed, Nonlinear vibrations control of a contact-mode AFM model via a time-delayed positive position Feedback, Alexandria Engineering Journal, 60, 963977(2021).

[34] Y.S. Hamed, A. El Shehry, M. Sayed, Nonlinear modified positive position feedback control of cantilever beam system carrying an intermediate lumped mass, Alexandria Engineering Journal ,59, 3847-3862(2020)

[35] Slotine, J.-J.E., Li,W.: Applied Nonlinear Control. Prentice Hall Englewood Cliffs (1991).

[36] Liang LH, Zhao P, Zhang ST, Yuan J, Wen Y. Simulation and analysis of Magnus rotating roll stabilizer at low speed. Ocean Engineering. (142) 491-500 (2017)

\section{Appendix}

$$
\begin{aligned}
& \xi_{11}=\frac{\partial \dot{a}_{1}}{\partial a_{1}}=-\frac{\mu_{1}}{2}+\frac{\alpha_{1} a_{2} \sin \left(\theta_{2}\right)}{4 \omega_{1}}-\frac{9 G_{1} \omega_{1}^{2} a_{1}^{2}}{8}+\frac{F_{1} \sin \left(\theta_{1}\right)}{4 \omega_{1}} \\
& \xi_{12}=\frac{\partial \dot{a}_{1}}{\partial \theta_{1}}=\frac{F_{1} a_{1} \cos \left(\theta_{1}\right)}{4 \omega_{1}}, \xi_{13}=\frac{\partial \dot{a}_{1}}{\partial a_{2}}=\frac{\alpha_{1} a_{1} \sin \left(\theta_{2}\right)}{4 \omega_{1}} \\
& \xi_{14}=\frac{\partial \dot{a}_{1}}{\partial \theta_{2}}=\frac{\alpha_{1} a_{1} a_{2} \cos \left(\theta_{2}\right)}{4 \omega_{1}}
\end{aligned}
$$


$\xi_{21}=\frac{\partial \dot{\theta}_{1}}{\partial a_{1}}=\frac{\sigma_{1}}{a_{1}}+\frac{\alpha_{1} a_{2} \cos \left(\theta_{2}\right)}{2 \omega_{1} a_{1}}$

$+\frac{\alpha_{2} \omega_{2}^{2} a_{2}^{2}}{4 \omega_{1} a_{1}}+\frac{F_{1} \cos \left(\theta_{1}\right)}{2 a_{1} \omega_{1}}, \xi_{22}=\frac{\partial \dot{\theta}_{1}}{\partial \theta_{1}}=-\frac{F_{1} \sin \left(\theta_{1}\right)}{2 \omega_{1}}$,

$\xi_{23}=\frac{1}{2 \omega_{1}}\left(\alpha_{1} \cos \left(\theta_{2}\right)+\alpha_{2} \omega_{2}^{2} a_{2}\right)$,

$\xi_{24}=-\frac{a_{2} \alpha_{1} \sin \left(\theta_{2}\right)}{2 \omega_{1}}$,

$\xi_{31}=\frac{\partial \dot{a}_{2}}{\partial a_{1}}=-\frac{a_{1} \delta_{1} \sin \left(\theta_{2}\right)}{2 \omega_{1}}$,

$\xi_{32}=\frac{\partial \dot{a}_{2}}{\partial \theta_{1}}=0, \quad \xi_{33}=\frac{\partial \dot{a}_{2}}{\partial a_{2}}=-\frac{\mu_{2}}{2}$,

$\xi_{34}=\frac{\partial \dot{a}_{2}}{\partial \theta_{2}}=-\frac{\delta_{1} a_{1}^{2} \cos \left(\theta_{2}\right)}{4 \omega_{1}}$,

$\xi_{41}=\frac{\partial \dot{\theta}_{2}}{\partial a_{1}}=\xi_{21}-\frac{\delta_{1} a_{1} \cos \left(\theta_{2}\right)}{2}$,

$\xi_{42}=\frac{\partial \dot{\theta}_{2}}{\partial \theta_{1}}=\xi_{22}, \xi_{43}=\frac{\partial \dot{\theta}_{2}}{\partial a_{2}}=\xi_{23}+\frac{\sigma_{2}-\sigma_{1}}{a_{2}}$,

$\xi_{44}=\frac{\partial \dot{\theta}_{2}}{\partial \theta_{2}}=\xi_{24}+\frac{\delta_{1} a_{1}^{2} \sin \left(\theta_{2}\right)}{4 \omega_{1} a_{2}}$.

$\eta_{1}=-\left(\xi_{11}+\xi_{22}+\xi_{33}+\xi_{44}\right)$

$\eta_{2}=-\left(\xi_{12} \xi_{21}+\xi_{31} \xi_{13}+\xi_{34} \xi_{43}+\xi_{42} \xi_{24}+\xi_{41} \xi_{14}\right)$

$+\xi_{11} \xi_{22}+\xi_{11} \xi_{33}+\xi_{22} \xi_{33}+\xi_{11} \xi_{44}$

$+\xi_{22} \xi_{44}+\xi_{33} \xi_{44}$

$\eta_{3}=\xi_{41} \xi_{14} \xi_{33}+\xi_{41} \xi_{14} \xi_{22}+\xi_{42} \xi_{24} \xi_{33}+\xi_{42} \xi_{24} \xi_{11}$

$+\xi_{43} \xi_{34} \xi_{22}+\xi_{43} \xi_{34} \xi_{11}+\xi_{44} \xi_{31} \xi_{13}+\xi_{44} \xi_{21} \xi_{12}$

$+\xi_{22} \xi_{31} \xi_{13}+\xi_{33} \xi_{21} \xi_{12}-\left(\xi_{44} \xi_{33} \xi_{22}+\xi_{44} \xi_{33} \xi_{11}\right.$

$+\xi_{44} \xi_{22} \xi_{11}+\xi_{41} \xi_{22} \xi_{24}+\xi_{41} \xi_{13} \xi_{34}+\xi_{42} \xi_{21} \xi_{14}$

$\left.+\xi_{42} \xi_{23} \xi_{34}+\xi_{43} \xi_{31} \xi_{14}+\xi_{31} \xi_{12} \xi_{23}+\xi_{11} \xi_{22} \xi_{33}\right)$

$\eta_{4}=\xi_{41} \xi_{12} \xi_{24} \xi_{33}+\xi_{41} \xi_{13} \xi_{34} \xi_{22}+\xi_{42} \xi_{21} \xi_{14} \xi_{33}$

$+\xi_{42} \xi_{23} \xi_{34} \xi_{11}+\xi_{43} \xi_{31} \xi_{14} \xi_{22}+\xi_{42} \xi_{24} \xi_{31} \xi_{13}$

$+\xi_{43} \xi_{34} \xi_{21} \xi_{12}+\xi_{44} \xi_{31} \xi_{12} \xi_{23}+\xi_{44} \xi_{33} \xi_{22} \xi_{11}$

$-\left(\xi_{41} \xi_{12} \xi_{23} \xi_{34}+\xi_{42} \xi_{21} \xi_{13} \xi_{34}+\xi_{42} \xi_{23} \xi_{31} \xi_{14}\right.$

$+\xi_{43} \xi_{31} \xi_{12} \xi_{24}+\xi_{41} \xi_{14} \xi_{33} \xi_{22}+\xi_{42} \xi_{24} \xi_{33} \xi_{11}$

$+\xi_{43} \xi_{34} \xi_{22} \xi_{11}+\xi_{44} \xi_{31} \xi_{13} \xi_{22}+\xi_{44} \xi_{33} \xi_{21} \xi_{12}$ 